\title{
Abnormal Liquid Chasing Effect in Paper Capillary Enables Versatile Gradient Generation on Microfluidic Paper Analytical Devices
}

Yu Liu ${ }^{1 \ddagger}$, Jin-Wen Shangguan ${ }^{1 \ddagger}, \mathrm{Bi}^{-} \mathrm{Yi} \mathrm{Xu}^{1,2^{*}}$, Xiao-Dong $\mathrm{Yu}^{1}$, Jing-Juan $\mathrm{Xu}^{1 *}$, HongYuan Chen ${ }^{1 *}$

${ }^{1}$ State Key Laboratory of Analytical Chemistry for Life Science and Collaborative Innovation Center of Chemistry for Life Sciences, School of Chemistry and Chemical Engineering, Nanjing University,

Nanjing 210023, China

${ }^{2}$ School of Engineering and Applied Sciences, Department of Physics, Harvard University, 29 Oxford Street, Cambridge, MA 02138, USA.

* Corresponding authors.

E-mail: xuby@nju.edu.cn, Tel./Fax: +86-25-89684862;

E-mail: xujj@nju.edu.cn, Tel./Fax: +86-25-89687294;

E-mail: hychen@nju.edu.cn, Tel./Fax: +86-25-89684862.

\section{Table of Contents:}

Contents

Cover page and table of contents

Design of the paper capillary chip

Colorimetry of BSA with different concentrations

RGB profiling of $1 \mathrm{D}$ gradient on $1 \mathrm{D}$ chips

Liquid transportation under harsh conditions

Time serial colorimetric analysis of gradient generation process on the paper capillary chip 


\section{Design of the paper capillary chip}

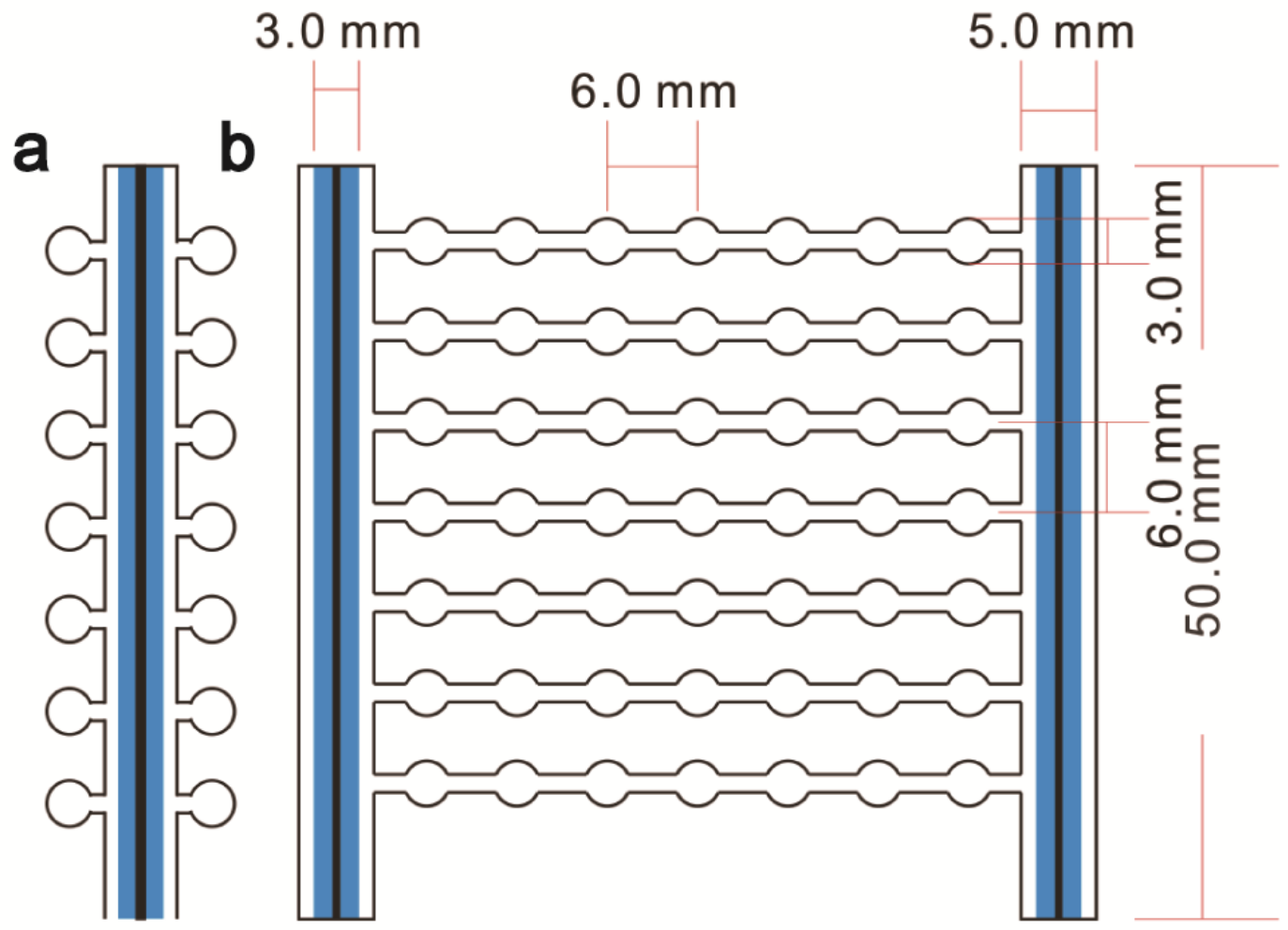

Figure S1. Design of single paper capillary chip and arrayed chip: a. single paper capillary chip; b. arrayed chip.

We designed paper capillary chips as Figure S1. The single paper capillary chip (Figure S1a) consists of a central trunk and test pads on both sides. The trunk was $5.0 \mathrm{~mm}$ width and $50.0 \mathrm{~mm}$ length. A groove with $0.5 \mathrm{~mm}$ for width was carved on the trunk, and a slice of tape with $3.0 \mathrm{~mm}$ (the blue in Figure S1) was attached to cover the groove, forming a paper capillary. There were two rows of test pads on both sides, 7 test pads on each side. Each test pad was connected with the trunk through $1.2 \mathrm{~mm}$ wide paper. The diameter of the test pads was $3.0 \mathrm{~mm}$, and the distance between them was $6.0 \mathrm{~mm}$. The arrayed chip (Figure S1b) consists of two trunks on both sides and a $7 * 7$ test pad array between them. The size of trunks and test pads on the arrayed chip was the same as the size on the single paper capillary chip. In the $7 * 7$ test pad array, each test pad on the same column was connected with each other through $1.2 \mathrm{~mm}$ wide paper. The chips were designed by CorelDraw X4 software. 


\section{Colorimetry of BSA with different concentrations}

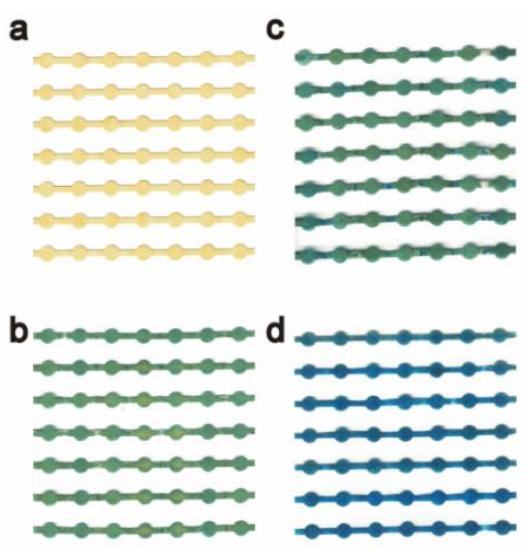

Figure S2. Colorimetry sample images of different concentrations of BSA, with $6 \mathrm{mM}$ TBPB: a. control (0 mg/mL BSA); b. $40 \mathrm{mg} / \mathrm{mL}$ BSA; c. $100 \mathrm{mg} / \mathrm{mL}$ BSA; d. $250 \mathrm{mg} / \mathrm{mL}$ BSA

We collected colorimetry images of different concentrations of BSA. The color of the original test pads was light yellow, the original color of TBPB. As BSA concentration increasing, the color gradually changes, from yellow (Figure $\mathrm{S} 2 \mathrm{a}, 0 \mathrm{mg} / \mathrm{mL}$ BSA) to green (Figure $\mathrm{s} 2 \mathrm{~b}, 40 \mathrm{mg} / \mathrm{mL} \mathrm{BSA}$; Figure S2c, $100 \mathrm{mg} / \mathrm{mL} \mathrm{BSA}$ ), and finally to blue (Figure $\mathrm{S} 2 \mathrm{~d}, 250 \mathrm{mg} / \mathrm{mL} \mathrm{BSA}$ ). Thus, it is safe to deduce that in the range of 0 to $100 \mathrm{mg} / \mathrm{mL}$ concentrations, the colorimetric reaction of TBPB is not saturated, and the color intensity of the sample can reflect the homogeneity of the sample distribution.

\section{RGB profiling of 1D gradient on 1D chips}

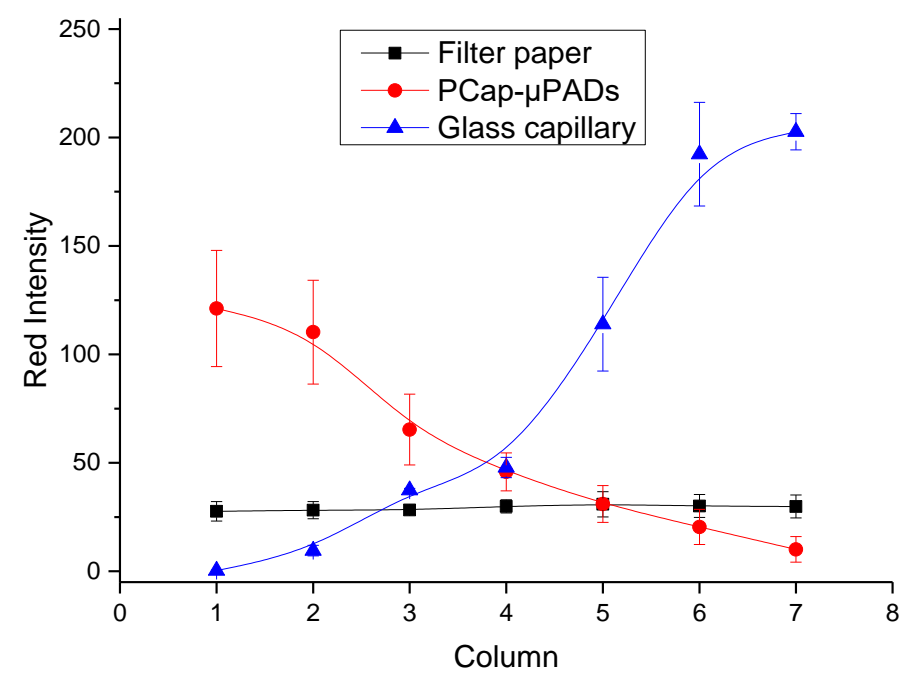

Figure S3. Red colorimetric analysis of gradient on glass capillary, single layer filter paper, and paper capillary chip (PCap- $\mu$ PADs). 
In the RGB coding system, white corresponds to $\operatorname{RGB}(255,255,255)$ while cyan corresponds to RGB $(0,255,255)$, magenta corresponds to RGB $(255,0,255)$, yellow corresponds to RGB (255, $255,0)$. The red intensity gradually decreases during the transition from red to blue. The test pads were named column 1-7 from bottom to top. In the glass capillary, the red intensity gradually increased from column1 to column7, indicated that the gradient from blue to red was generated in a glass capillary. On the filter paper, the distribution of red intensity was relatively uniform. So there was no obvious solution gradient on the filter paper. On the paper capillary chip, the red intensity gradually decreased from column1 to column7, indicated that the solution gradient was also generated on the paper capillary chip. The gradient on the paper capillary chip was opposite to the gradient in the glass capillary, which is caused by the abnormal liquid chasing effect.

\section{Liquid transportation under harsh conditions}

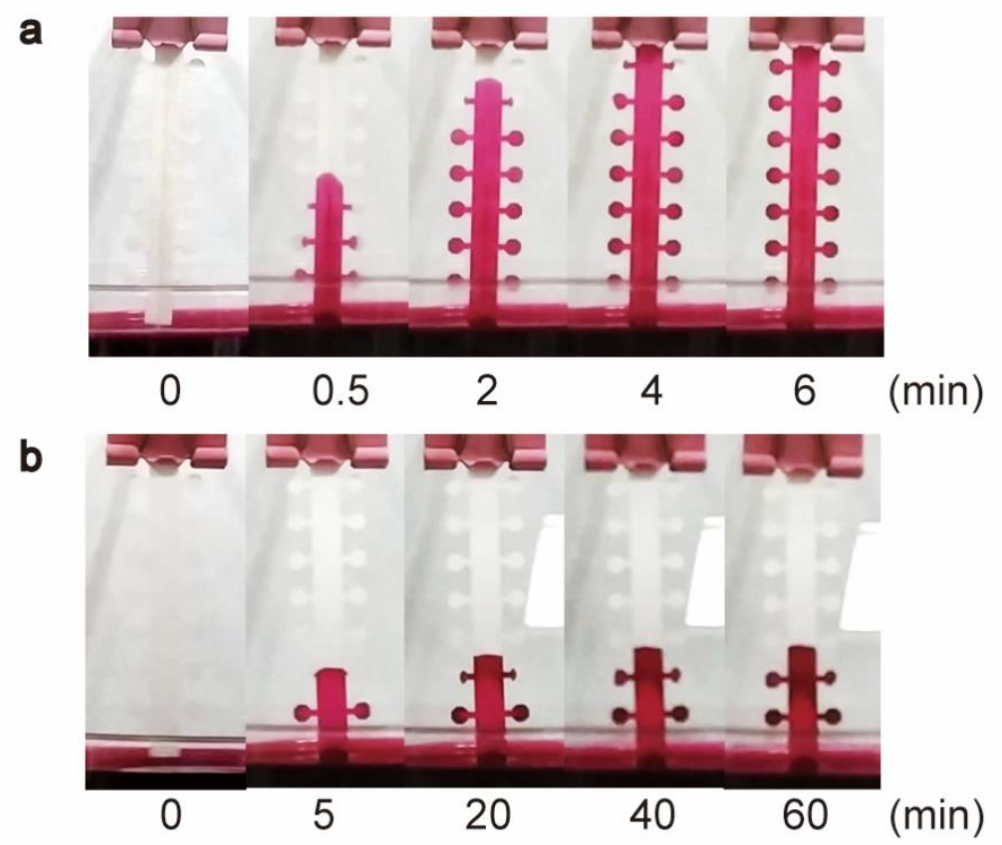

Figure S4. Time serial images of liquid transportation on (a) a paper capillary chip and (b) a single piece of paper under harsh condition (in an incubator with the temperature set to $50^{\circ} \mathrm{C}$ and dried with phosphorus pentoxide).

To evaluate the liquid transportation ability of the paper capillary chip under harsh conditions, the whole sampling process was carried out in an incubator with the temperature set to $50^{\circ} \mathrm{C}$ and the air-dried with phosphorus pentoxide. The liquid transportation process was recorded in figure S4a. It shows, the chip still managed to transport the samples to all the test pads, but took doubled time compared with that in the normal condition $\left(20^{\circ} \mathrm{C}, 50 \%\right.$ humidity). We also tested the liquid transportation in a single piece of paper as shown in figure S4b. Even over $1 \mathrm{~h}$, the sample cannot reach the third column of the test pads, indicating, it is impossible to carry out such sampling with only a single piece of paper. 


\section{Time serial colorimetric analysis of gradient generation process on}

\section{the paper capillary chip}
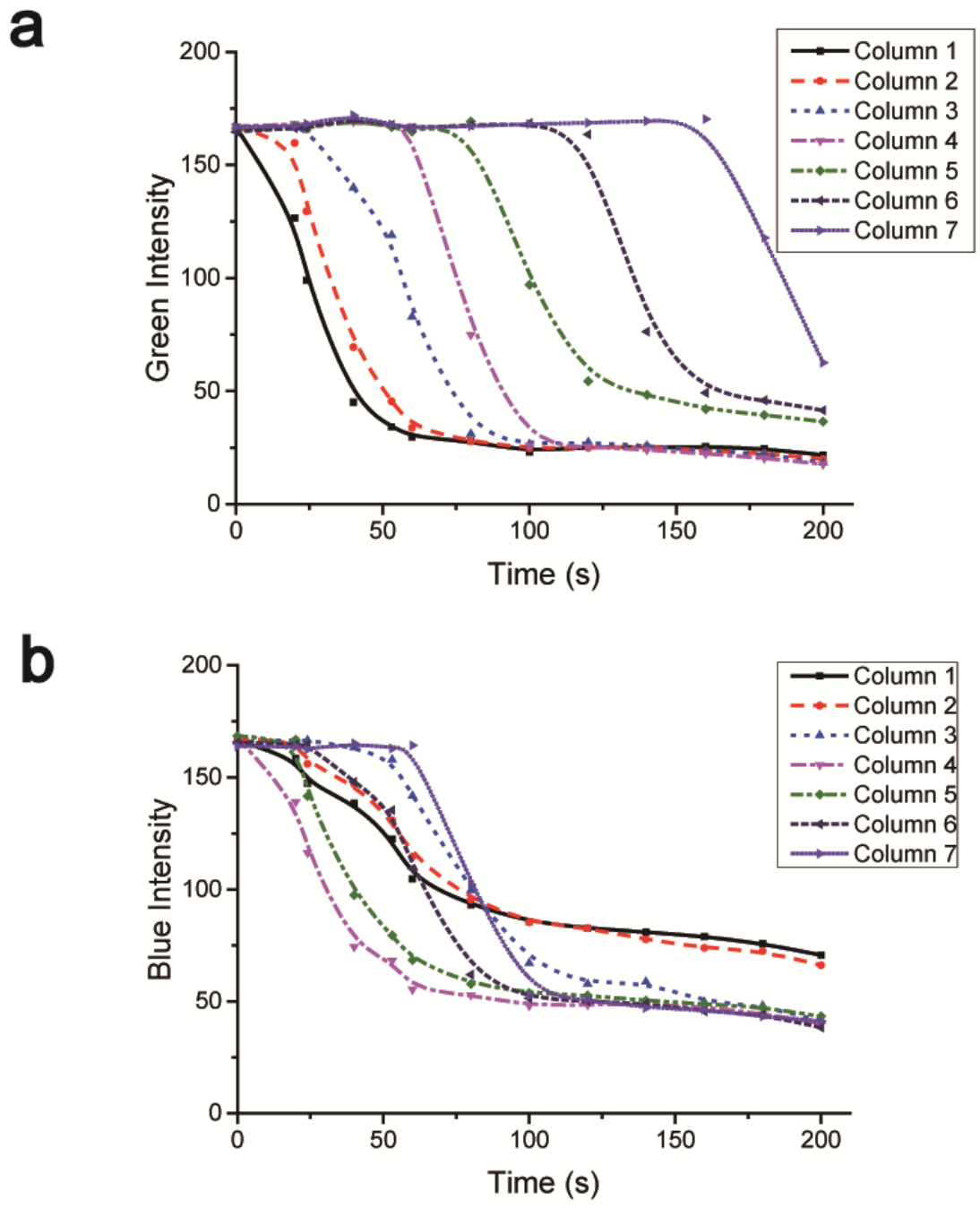

Figure S5. Time serial colorimetric analysis of test pads of the paper capillary chip: a. green color intensity analysis, b. blue color intensity analysis.

Besides red intensity, we also traced the green and blue intensity in the paper capillary region at different heights correspond to each column of the chip. For green color intensity (Figure S5a), the white color holds the highest reading, the blue color is smaller, and the red color is the smallest. For blue intensity (Figure S5b), the white color holds the highest reading, too. But the blue ink was smaller than the red ink, caused the color of red ink did not exactly match the red condition in RGB, but closer to magenta $(255,0,255)$. A sharp decrease in color intensity indicates that the ink enters the corresponding measurement position. In the measurement result, the color intensity on the higher position decreased later, which was consistent with the infiltration order of the solution. At the last time (200 s), the green intensity gradually decreased from top to bottom, while the blue color increased. The analysis results of the green and blue color intensity were consistent with the analysis of red intensity (Fig 2f). 